\title{
Consistent forcing scheme in the cascaded lattice Boltzmann method
}

\author{
Linlin Fei ${ }^{1}$ and Kai Hong Luo ${ }^{1,2, *}$ \\ ${ }^{1}$ Center for Combustion Energy, Key laboratory for Thermal Science and Power Engineering of Ministry of Education, \\ Department of Thermal Engineering, Tsinghua University, Beijing 100084, China \\ ${ }^{2}$ Department of Mechanical Engineering, University College London, Torrington Place, London WC1E 7JE, UK
}

(Received 31 May 2017; published 13 November 2017)

\begin{abstract}
In this paper, we give an alternative derivation for the cascaded lattice Boltzmann method (CLBM) within a general multiple-relaxation-time (MRT) framework by introducing a shift matrix. When the shift matrix is a unit matrix, the CLBM degrades into an MRT LBM. Based on this, a consistent forcing scheme is developed for the CLBM. The consistency of the nonslip rule, the second-order convergence rate in space, and the property of isotropy for the consistent forcing scheme is demonstrated through numerical simulations of several canonical problems. Several existing forcing schemes previously used in the CLBM are also examined. The study clarifies the relation between MRT LBM and CLBM under a general framework.
\end{abstract}

DOI: 10.1103/PhysRevE.96.053307

\section{INTRODUCTION}

The lattice Boltzmann method (LBM), based on the simplified kinetic models, has gained remarkable success for numerical simulations of complex fluid flows and beyond, with applications to (but not limited to) microflows, flows in porous media, turbulence, multiphase flows, and reactive flows [1-9]. The LBM solves a specific discrete Boltzmann equation for distribution functions, designed to recover the Navier-Stokes (N-S) equations in the macroscopic limit. The mesoscale nature of LBM allows its natural incorporation of micro and mesoscale physics, while the highly efficient algorithm makes it affordable computationally [9].

In the standard "collision-streaming" LBM algorithm, the simplest collision operator is the Bhatnagar-Gross-Krook (BGK) or single-relaxation-time (SRT) operator, in which all the distribution functions relax at an identical rate to their local equilibrium counterparts and the relaxation rate is related to the kinematic viscosity [10]. The multiple-relaxation-time (MRT) operator is another extensively used operator [11], in which the collision is executed in the raw moment space and the relaxation rates for different moments can be different. More recently, a central-moment-based or cascaded operator was proposed by Geier et al. [12]. In the cascaded lattice Boltzmann method (CLBM), the collision is carried out in the space of central moment rather than that of raw moment as in the MRT LBM. Compared with the BGK operator, the MRT and cascaded operators can enhance the numerical stability significantly [12-15]. Although the collision steps in these LBMs are quite different, the streaming steps are carried out in the same way by streaming the post-collision distributions to their neighbors. It should be noted that other collision operators, such as the two-relaxation-time (TRT) operator $[16,17]$ and the entropic operator $[18,19]$, are also very popular in the lattice Boltzmann community.

In many fluid systems, an external or internal force field plays an important role in the flow behaviors. To incorporate the forcing effect, different forcing treatments or forcing schemes have been proposed in the previous literatures

*k.luo@ucl.ac.uk
[4,20-24]. In 2002, Guo et al. analyzed the discrete lattice effects on the forcing scheme and developed a representation of the forcing term [25]. Guo et al. then extended the method to the MRT LBM in 2008 [26]. For the CLBM, there is still no commonly used forcing scheme, while one scheme by method of central moments has been proposed by Premnath et al. [27]. In Ref. [28], Lycett-Brown and Luo adopted the forcing scheme for the BGK LBM in the CLBM directly. As analyzed by De Rosis [29], the method proposed by Premnath et al. may encounter cumbersome practical implementations. Based on the central moments of a discrete equilibrium, a forcing scheme has been developed in Ref. [29].

However, there is still no analysis about whether these forcing schemes in the CLBM are consistent with the extensively used forcing schemes in the MRT LBM [26] and BGK LBM [25]. In this paper, we propose an alternative derivation for the CLBM by introducing a shift matrix. This approach clarifies the relationship between the MRT LBM and CLBM. Based on this framework, we present a consistent forcing scheme in CLBM and show that the previous methods in Refs. [27-29] are not consistent. The rest of the paper is structured as follows. Section II gives the new derivation for the CLBM and presents the consistent forcing scheme. Section III presents theoretical analyses for the previous forcing schemes. Numerical verifications are presented in Sec. IV. Finally, conclusions are made in Sec. V.

\section{CLBM AND CONSISTENT FORCING SCHEME}

\section{A. Cascaded LBM}

Without losing generality, the D2Q9 lattice [10] is adopted here. The lattice speed $c=\Delta x / \Delta t=1$ and the lattice sound speed $c_{s}=1 / \sqrt{3}$ are adopted, where $\Delta x$ and $\Delta t$ are the lattice space and time steps. The discrete velocities $\mathbf{e}_{i}=\left[\left|e_{i x}\right\rangle,\left|e_{i y}\right\rangle\right]$ are defined as

$$
\begin{aligned}
& \left|e_{i x}\right\rangle=[0,1,0,-1,0,1,-1,-1,1]^{\top}, \\
& \left|e_{i y}\right\rangle=[0,0,1,0,-1,1,1,-1,-1]^{\top},
\end{aligned}
$$

where $i=0 \ldots 8,|\cdot\rangle$ denotes a nine-dimensional column vector, and the superscript $T$ denotes the transposition.

Here we propose a new derivation for the CLBM, which is different from and more intelligible than that given by Geier 
et al. [12]. We first define the raw and central velocity moments of the discrete distribution functions (DFs) $f_{i}$,

$k_{m n}=\left\langle f_{i} \mid e_{i x}^{m} e_{i y}^{n}\right\rangle, \quad \tilde{k}_{m n}=\left\langle f_{i} \mid\left(e_{i x}-u_{x}\right)^{m}\left(e_{i y}-u_{y}\right)^{n}\right\rangle$,

where $u_{x}$ and $u_{y}$ are the horizontal and vertical velocity components. The equilibrium counterparts $k_{m n}^{\mathrm{eq}}$ and $\tilde{k}_{m n}^{\mathrm{eq}}$ are defined analogously by replacing $f_{i}$ with the discrete equilibrium distribution functions (EDFs) $f_{i}^{\text {eq }}$ in Eq. (2). In the previous CLBM, the recombined raw moments are adopted,

$$
\left|T_{i}\right\rangle=\left[k_{00}, k_{10}, k_{01}, k_{20}+k_{02}, k_{20}-k_{02}, k_{11}, k_{21}, k_{12}, k_{22}\right]^{\top},
$$

so do the recombined central moments $\tilde{T}_{i}$. The transformation from the discrete DFs to their raw moments can be performed through a transformation matrix $\mathbf{M}$, and the shift from the raw moments to central moments can be performed though a shift matrix $\mathbf{N}$,

$$
\left|T_{i}\right\rangle=\mathbf{M}\left|f_{i}\right\rangle, \quad\left|\tilde{T}_{i}\right\rangle=\mathbf{N}\left|T_{i}\right\rangle
$$

$$
\mathbf{N}=\left[\begin{array}{cccc}
1 & 0 & 0 & 0 \\
-u_{x} & 1 & 0 & 0 \\
-u_{y} & 0 & 1 & 0 \\
u_{x}^{2}+u_{y}^{2} & -2 u_{x} & -2 u_{y} & 1 \\
u_{x}^{2}-u_{y}^{2} & -2 u_{x} & 2 u_{y} & 0 \\
u_{x} u_{y} & -u_{y} & -u_{x} & 0 \\
-u_{x}^{2} u_{y} & 2 u_{x} u_{y} & u_{x}^{2} & -u_{y} / 2 \\
-u_{y}^{2} u_{x} & u_{y}^{2} & 2 u_{x} u_{y} & -u_{x} / 2 \\
u_{x}^{2} u_{y}^{2} & -2 u_{x} u_{y}^{2} & -2 u_{y} u_{x}^{2} & u_{x}^{2} / 2+u_{y}^{2} / 2
\end{array}\right.
$$

$\mathbf{N}=\left[\begin{array}{cccc}1 & 0 & 0 & 0 \\ -u_{x} & 1 & 0 & 0 \\ -u_{y} & 0 & 1 & 0 \\ u_{x}^{2}+u_{y}^{2} & -2 u_{x} & -2 u_{y} & 1 \\ u_{x}^{2}-u_{y}^{2} & -2 u_{x} & 2 u_{y} & 0 \\ u_{x} u_{y} & -u_{y} & -u_{x} & 0 \\ -u_{x}^{2} u_{y} & 2 u_{x} u_{y} & u_{x}^{2} & -u_{y} / 2 \\ -u_{y}^{2} u_{x} & u_{y}^{2} & 2 u_{x} u_{y} & -u_{x} / 2 \\ u_{x}^{2} u_{y}^{2} & -2 u_{x} u_{y}^{2} & -2 u_{y} u_{x}^{2} & u_{x}^{2} / 2+u_{y}^{2} / 2\end{array}\right.$

In the collision step for CLBM, the central moments $\tilde{T}_{i}$ are relaxed to their equilibrium values $\tilde{T}_{i}^{\mathrm{eq}}$. Thus, the post-collision central moments are

$$
\begin{aligned}
\left|\tilde{T}_{i}^{*}\right\rangle & =(\mathbf{I}-\mathbf{S})\left|\tilde{T}_{i}\right\rangle+\mathbf{S}\left|\tilde{T}_{i}^{\mathrm{eq}}\right\rangle \\
& =(\mathbf{I}-\mathbf{S}) \mathbf{N M}\left|f_{i}\right\rangle+\mathbf{S N M}\left|f_{i}^{\mathrm{eq}}\right\rangle,
\end{aligned}
$$

where $\mathbf{S}=\operatorname{diag}\left(s_{0}, s_{1}, s_{1}, s_{b}, s_{2}, s_{2}, s_{3}, s_{3}, s_{4}\right)$ is a diagonal relaxation matrix. The kinematic and bulk viscosities are related to the relaxation parameters $s_{2}=1 /(3 v+0.5)$ and $s_{b}=1 /(3 \xi+$ $0.5)$, respectively. As recommended in Refs. [12,27,28], the central moments of $f_{i}^{\mathrm{eq}}$ are set equal to the continuous central moments of the Maxwell-Boltzmann distribution in continuous velocity space. To be specific,

$$
\left|\tilde{T}_{i}^{\mathrm{eq}}\right\rangle=\left[\rho, 0,0,2 \rho c_{s}^{2}, 0,0,0,0, \rho c_{s}^{4}\right]^{\top},
$$

where $\rho$ is the fluid density, thus the matrix manipulation is not needed for $f_{i}^{\mathrm{eq}}$. The corresponding discrete EDF is in fact a generalized local equilibrium [27,30]. Due to the definitions of the transformation and shift matrices, both of them are invertible (explicit expressions for $\mathbf{M}^{-1}$ and $\mathbf{N}^{-1}$ are given in the Appendix). The post-collision discrete DFs are given by

$$
\left|f_{i}^{*}\right\rangle=\mathbf{M}^{-1} \mathbf{N}^{-1}\left|\tilde{T}_{i}^{*}\right\rangle
$$

The formulations for $\mathbf{M}$ and $\mathbf{N}$ depend on the considered rawmoment set in Eq. (3), which are not unique. In the present study, the transformation matrix $\mathbf{M}$ is expressed as [28]

$$
\mathbf{M}=\left[\begin{array}{rrrrrrrrr}
1 & 1 & 1 & 1 & 1 & 1 & 1 & 1 & 1 \\
0 & 1 & 0 & -1 & 0 & 1 & -1 & -1 & 1 \\
0 & 0 & 1 & 0 & -1 & 1 & 1 & -1 & -1 \\
0 & 1 & 1 & 1 & 1 & 2 & 2 & 2 & 2 \\
0 & 1 & -1 & 1 & -1 & 0 & 0 & 0 & 0 \\
0 & 0 & 0 & 0 & 0 & 1 & -1 & 1 & -1 \\
0 & 0 & 0 & 0 & 0 & 1 & 1 & -1 & -1 \\
0 & 0 & 0 & 0 & 0 & 1 & -1 & -1 & 1 \\
0 & 0 & 0 & 0 & 0 & 1 & 1 & 1 & 1
\end{array}\right] .
$$

Asinari proposed a "shift matrix," where the focus was on the relation between the cascaded operator and TRT operator [30]. Another raw-moment set $\left|T_{i}\right\rangle=\left[k_{00}, k_{10}, k_{01}, k_{20}, k_{02}, k_{11}, k_{21}, k_{12}, k_{22}\right]^{\top}$ was adopted in Ref. [30], leading to a different formulation for the "shift matrix" in Ref. [30]. In this paper, the formulation for $\mathbf{N}$ is

$\left.\begin{array}{ccccc}0 & 0 & 0 & 0 & 0 \\ 0 & 0 & 0 & 0 & 0 \\ 0 & 0 & 0 & 0 & 0 \\ 0 & 0 & 0 & 0 & 0 \\ 1 & 0 & 0 & 0 & 0 \\ 0 & 1 & 0 & 0 & 0 \\ -u_{y} / 2 & -2 u_{x} & 1 & 0 & 0 \\ u_{x} / 2 & -2 u_{y} & 0 & 1 & 0 \\ u_{y}^{2} / 2-u_{x}^{2} / 2 & 4 u_{x} u_{y} & -2 u_{y} & -2 u_{x} & 1\end{array}\right]$.

In the streaming step, the post-collision discrete DFs in space $\mathbf{x}$ stream to the neighbor nodes $\left(\mathbf{x}+\mathbf{e}_{i} \Delta t\right)$ along the discrete velocity direction as usual $[25,26,31]$,

$$
f_{i}\left(\mathbf{x}+\mathbf{e}_{i} \Delta t, t+\Delta t\right)=f_{i}^{*}(\mathbf{x}, t)
$$

Using the Chapman-Enskog analysis, the incompressible N-S equations can be reproduced in the low-Mach number limit $[27,28]$. The hydrodynamics variables are obtained as

$$
\rho=\sum_{i} f_{i}, \quad \rho \mathbf{u}=\sum_{i} f_{i} \mathbf{e}_{i} .
$$

It can be readily shown that when the shift matrix $\mathbf{N}$ is a unit matrix, the CLBM degrades into a nonorthogonal MRT LBM as in Refs. [28,31]. Thus, within a general multiplerelaxation-time framework, the present derivation clarifies the relationship between the MRT LBM and CLBM.

\section{B. Consistent forcing scheme}

Inspired by the method proposed by He et al. [23,24], to incorporate an external or internal force field $\mathbf{F}=\left[F_{x}, F_{y}\right]$ into the CLBM with a high-order accuracy, a second-order trapezoidal scheme is adopted. Thus, the evolution equation 
for central moments can be given as

$$
\begin{aligned}
& \left|\tilde{T}_{i}\left(\mathbf{x}+\mathbf{e}_{i} \Delta t, t+\Delta t\right)\right\rangle=(\mathbf{I}-\mathbf{S})\left|\tilde{T}_{i}(\mathbf{x}, t)\right\rangle+\mathbf{S} \mid \tilde{T}_{i}^{\mathrm{eq}}(\mathbf{x}, t) \\
& \quad+\Delta t / 2\left|C_{i}(\mathbf{x}, t)+C_{i}\left(\mathbf{x}+\mathbf{e}_{i} \Delta t, t+\Delta t\right)\right\rangle
\end{aligned}
$$

where $C_{i}$ are central moments of the forcing terms $R_{i}$. According to the assumption by He et al. [23,24], $R_{i}$ can be given by

$$
R_{i}=\frac{\mathbf{F}}{\rho} \frac{\left(\mathbf{e}_{i}-\mathbf{u}\right)}{c_{s}^{2}} f_{i}^{\mathrm{eq}} .
$$

When using the generalized local equilibrium, $C_{i}$ can be obtained explicitly as

$$
\left|C_{i}\right\rangle=\mathbf{N M}\left|R_{i}\right\rangle=\left[0, F_{x}, F_{y}, 0,0,0, c_{s}^{2} F_{y}, c_{s}^{2} F_{x}, 0\right]^{\top} .
$$

In the practical implementation, the following transformation method is adopted to remove the implicitness in Eq. (12),

$$
\bar{f}_{i}=f_{i}-1 / 2 R_{i}, \quad \bar{T}_{i}=\tilde{T}_{i}-1 / 2 C_{i} .
$$

By substituting Eq. (15) into Eq. (12), the collision step in central moments is modified by

$$
\begin{aligned}
\left|\bar{T}_{i}^{*}\right\rangle & =(\mathbf{I}-\mathbf{S}) \mathbf{N M}\left|\bar{f}_{i}\right\rangle+\mathbf{S N M}\left|f_{i}^{\mathrm{eq}}\right\rangle+(\mathbf{I}-\mathbf{S} / 2) \mathbf{N M}\left|R_{i}\right\rangle \\
& =(\mathbf{I}-\mathbf{S})\left|\bar{T}_{i}\right\rangle+\mathbf{S}\left|\tilde{T}_{i}^{\mathrm{eq}}\right\rangle+(\mathbf{I}-\mathbf{S} / 2)\left|C_{i}\right\rangle .
\end{aligned}
$$

By using $\left|\bar{f}_{i}^{*}\right\rangle=\mathbf{M}^{-1} \mathbf{N}^{-1}\left|\bar{T}_{i}^{*}\right\rangle$, the streaming step can be executed as usual,

$$
\bar{f}_{i}\left(\mathbf{x}+\mathbf{e}_{i} \Delta t, t+\Delta t\right)=\bar{f}_{i}^{*}(\mathbf{x}, t) .
$$

Then the fluid velocity is redefined by

$$
\rho \mathbf{u}=\sum_{i} \bar{f}_{i} \mathbf{e}_{i}+\Delta t \mathbf{F} / 2 .
$$

Remark 1. In Eq. (12), the forcing terms in the present scheme are considered by means of central moments, which is compatible with the basic ideology (collision in the centralmoment space) in the CLBM.

Remark 2. When the shift matrix $\mathbf{N}$ in Eq. (16) is a unit matrix, the CLBM with the present forcing scheme will degrade into the MRT LBM proposed by Liu et al. [31] with some high-order terms. It is known that the method of Liu et al. [31] is equivalent to the method of Guo et al. in Ref. [26].

Remark 3. In the original forcing scheme proposed by Guo et al. [25], the forcing terms are defined as $R_{G i}=$ $w_{i}\left[\left(\mathbf{e}_{i}-\mathbf{u}\right) / c_{s}^{2}+\left(\mathbf{e}_{i} \cdot \mathbf{u}\right) \mathbf{e}_{i} / c_{s}^{4}\right] \mathbf{F}$. It is easy to find that the forcing terms in Eq. (13) are equivalent to $R_{G i}$ plus some high-order terms. The constraint conditions for the forcing terms (see Eq. (7) in Ref. [25]) are also satisfied in the present scheme. In particular, if all the parameters in the matrix $\mathbf{S}$ are set equal to $s_{2}$, the CLBM with the present forcing scheme degrades into the BGK LBM with a generalized local equilibrium and the forcing scheme by Guo et al.

Remark 4. It is also found (see Sec. IV) that the zero-slip velocity boundary condition for the half-way bounce-back rule $\left[s_{3}=\left(16-8 s_{2}\right) /\left(8-s_{2}\right)\right]$ discussed in Refs. [26,32] is also applicable to the present forcing scheme.

From the above reasons, we proclaim the present forcing scheme as a consistent scheme in the CLBM.

\section{OTHER FORCING SCHEMES}

In this section, several existing methods to incorporate forcing terms into the CLBM in the literature are summarized. To show the inconsistencies in these forcing schemes, they are all written in the general multiple-relaxation-time frame proposed in Sec. II.

\section{A. Forcing scheme by Premnath et al.}

In 2009, Premnath et al. [27] proposed a forcing scheme to incorporate forcing terms into CLBM. Inspired by $\mathrm{He}$ et al. [24], they proposed a change of continuous distribution function $f$ due to the presence of a force field,

$$
\Delta f=\frac{\mathbf{F}}{\rho} \cdot \frac{\left(\mathbf{e}_{i}-\mathbf{u}\right)}{c_{s}^{2}} f^{M},
$$

where $f^{M}$ is the Maxwell-Boltzmann distribution in continuous velocity space. The central moments of $\Delta f, C_{P i}$, are then incorporated into the collision stage by

$$
\left|\bar{T}_{i}^{*}\right\rangle=(\mathbf{I}-\mathbf{S})\left|\bar{T}_{i}\right\rangle+\mathbf{S}\left|\tilde{T}_{i}^{\mathrm{eq}}-C_{P i} / 2\right\rangle .
$$

The discrete counterparts for $\Delta f, \Delta f_{i}$ are also needed (see Eq.(37) in Ref. [27]) to obtain the post-collision discrete DFs,

$$
\left|\bar{f}_{i}^{*}\right\rangle=\mathbf{M}^{-1} \mathbf{N}^{-1}\left|\bar{T}_{i}^{*}\right\rangle+\left|\Delta f_{i}\right\rangle .
$$

The fluid velocity is defined as in Eq. (18).

It should be noted that the original derivation in Ref. [27] is tedious, and $C_{P i}$ and $\Delta f_{i}$ correspond to $\hat{\sigma}_{x^{m} y^{n}}$ and $S_{a}$ in Ref. [27], respectively. Although the method is compatible with the central-moment-based collision operator, the explicit formulations of $\Delta f_{i}$ and their raw moments are needed, which makes the practical implementations cumbersome [29]. Moreover, in their definition for $C_{P i}$, the high-order nonzero terms are removed arbitrarily,

$$
\left|C_{P i}\right\rangle=\mathbf{N M}\left|\Delta f_{i}\right\rangle=\left[0, F_{x}, F_{y}, 0,0,0,0,0,0\right]^{\top} .
$$

Although they think that the high-order terms do not affect consistency, we certainly see some inconsistencies. For example, the key elements in $\Delta f_{i}$ and $R_{G i}$ are apparently inconsistent,

$$
\begin{aligned}
& \Delta f_{0}=-2 F_{x} u_{x}-2 F_{y} u_{y}+O\left(u^{3}\right), \\
& R_{G 0}=-3 F_{x} u_{x}-3 F_{y} u_{y},
\end{aligned}
$$

which will affect numerical performances (see in Sec. IV).

\section{B. Forcing scheme by Lycett-Brown and Luo}

Cascaded LBM was first used to simulated multiphase flows by Lycett-Brown et al. [28]. In their method, three forcing schemes, the Shan-Chen method [4], the EDM method [33], and the Guo method [25], were adopted directly in the CLBM.

As discussed in the literature [34,35], both the Shan-Chen method and EDM method will lead to some additional terms in the recovered macroscopic equations. The additional terms may have some positive effects on the numerical performance of the Shan-Chen model [4], but it is not recommended to use the Shan-Chen method and EDM method in the CLBM directly for general flows. In the present work, we only consider the 
CLBM with the forcing scheme of Guo et al. Thus, the collision stage in central moments can be written as

$$
\left|\bar{T}_{i}^{*}\right\rangle=(\mathbf{I}-\mathbf{S})\left|\bar{T}_{i}\right\rangle+\mathbf{S}\left|\tilde{T}_{i}^{\mathrm{eq}}\right\rangle+\left(1-s_{2} / 2\right) \mathbf{N M}\left|R_{G i}\right\rangle,
$$

and the fluid velocity is also defined as in Eq. (18).

\section{Forcing scheme by De Rosis}

Recently, De Rosis proposed an alternative method to incorporate forcing terms into the CLBM. The collision stage in central moments is

$$
\left|\bar{T}_{i}^{*}\right\rangle=(\mathbf{I}-\mathbf{S})\left|\bar{T}_{i}\right\rangle+\mathbf{S}\left|\tilde{T}_{i}^{\mathrm{eq}}\right\rangle+\frac{1}{2}\left|\xi_{i}\right\rangle
$$

where $\xi_{i}$ are the central moments of the forcing terms, and the fluid velocity is also defined as in Eq. (18).

Unfortunately, there is a typographical error in Eq. (16) of the paper [29]. Particularly, the sign in front of $\xi_{i} / 2$ is not correct. In this method, the forcing terms are defined by the truncated local equilibrium DF, which leads to a lot of velocity terms in $\xi_{i}$ (see Eq. (15) in Ref. [29]). Due to the definition of central moments, it is not recommended to include velocity terms in $\xi_{i}$. Thus, there are some spurious effects using this method. It is also noted that the computational load for $\xi_{i}$ is much higher than that of $C_{i}$ in Eq. (14). Comparing Eq. (25) with Eq. (16), it is seen that the relaxation rate for each element of $\xi_{i}$ is 1.0 in this method, which is not consistent with the multiple-ralaxation-time methodology in the CLBM.

\section{Discussions}

Remark 5. In the CLBM, the first three central moments are conserved moments, corresponding to conservations of mass and momentum. Thus, the first two parameters $\left(s_{0}\right.$ and $\left.s_{1}\right)$ in the relaxation matrix $\mathbf{S}$ can be chosen freely. This property is retained in the present forcing scheme and the forcing scheme by Premnath $e t$ al. [27], because the relaxation matrix acts on the forcing terms in these two methods [see Eqs. (16) and (20)]. However, $s_{1}$ needs to be set equal to $s_{2}$ in the forcing scheme by Lycett-Brown and Luo [28] and to be 1.0 in the forcing scheme by De Rosis [29], to guarantee the conservation of momentum.

Remark 6. If $s_{3}$ is set to be 2.0 , the forcing effect on the thirdorder central moments in Eq. (16) is removed. Thus, the present scheme degrades into the forcing scheme by Premnath et al. [27] only when $s_{3}=2.0$. Similarly, only when $s_{3}=1.0$, the difference between the forcing scheme by De Rosis [29] and the present scheme can be removed. Finally, only in the BGK limitation when all the parameters are equal to $s_{2}$, the present scheme degrades into the scheme by Lycett-Brown and Luo [28]. In general, the deviations between the above three forcing schemes and the present consistent forcing scheme should be approximately proportional to $\left(s_{3}-2\right) / 2,\left(s_{3}-s_{2}\right) / 2$ and $\left(s_{3}-1\right) / 2$, respectively.

Remark 7. In 2015, an improved forcing scheme for the pseudopotential model [4] in multiphase flow was proposed by Lycett Brown and Luo [36]. The improved forcing scheme was then incorporated into the CLBM for multiphase flow with large-density-ratio at high Reynolds and Weber numbers [14]. The basic philosophy of the improved forcing scheme is introducing artificial terms in the pressure tensor to counteract the lack of thermodynamical consistency in the original pseudopotential model. Thus, it is not suitable for general flows with a force field. Besides, another simple forcing method was used in the CLBM to simulate turbulent channel flow in 2011 [37]. However, as analyzed by Guo et al. [25], the method used in Ref. [37] cannot recover the accurate macroscopic equations with a spatial and/or temporal variational force field in the BGK LBM, not to mention in the CLBM.

\section{NUMERICAL SIMULATIONS}

In this section, we conduct several benchmark cases to verify the consistent forcing scheme. The other three methods mentioned in Sec. III are also used to validate our arguments. The three methods [27-29] and the present method are denoted by $M_{1}, M_{2}, M_{3}$, and $M_{p}$, respectively. In the simulation, $s_{1}$ is set to be $s_{2}$ in $M_{2}$, but to be 1.0 in other methods.

\section{A. Steady Poiseuille flow}

The first problem considered is a steady Poiseuille flow driven by a constant body force $\mathbf{F}$. The flow direction is set to be the positive direction of the $x$ axial, thus $\mathbf{F}=\left[F_{x}, 0\right]$. The analytical solution for a channel of width $2 L$ is

$$
\mathbf{u}_{a}=\left[\frac{F_{x}}{2 v}\left(L^{2}-y^{2}\right), 0\right] .
$$

The periodic boundary conditions are used in the flow direction, while the standard half-way bounce-back boundary scheme is used for nonslip boundary conditions at the walls. Due to the simple flow configuration, the length of the channel is set to be $3 \Delta x$ to save the computational load.

As analyzed by previous researchers [26,32], when the relaxation rate for the energy flux is chosen to be $s_{3}=$ $\left(16-8 s_{2}\right) /\left(8-s_{2}\right)$, no numerical slip occurs in the Poiseuille flow for the MRT LBM. To check its applicability in the CLBM with the present forcing scheme, we first choose kinematic viscosity $v=0.5, F_{x}=0.01$, and only three nodes are used to cover the channel width $(2 L=3 \Delta x)$. We change $s_{3}$ from 0.2 to 1.8 with a 0.05 interval, and the other parameters are set equal to $s_{2}$. The residual error $E_{R}<1 \times 10^{-9}$ is used as the convergence criterion, and the relative error $E_{2}$ is calculated for the following analysis:

$$
E_{R}=\sqrt{\frac{\sum\left(\mathbf{u}_{(t+1000 \delta t)}-\mathbf{u}_{t}\right)^{2}}{\sum \mathbf{u}_{(t+1000 \delta t)}^{2}}}, \quad E_{2}=\sqrt{\frac{\sum\left(\mathbf{u}-\mathbf{u}_{a}\right)^{2}}{\sum \mathbf{u}_{a}^{2}}},
$$

where the summation operator $\sum$ is over all grid nodes. For this case, the needed value of $s_{3}$ for the nonslip rule is 1.6. As shown in Fig. 1, the relative error for each method changes with different values of $s_{3}$. But only in the present method, the minimum value of $E_{2}$ is achieved when $s_{3}=1.6$. And when the nonslip condition is satisfied, the relative error reaches an extremely small value even in a very coarse mesh.

To further confirm the consistent nonslip boundary condition in the present method, we conduct several other cases. Now the channel width is set to be $50 \Delta x$, and different body forces $F_{x}=\left[1 \times 10^{-6}, 3 \times 10^{-6}, 5 \times 10^{-6}, 7 \times 10^{-6}\right]$ are considered. The configurations are the same as those in Ref. [27], $s_{3}$ is chosen according to the nonslip rule, while other relaxation 


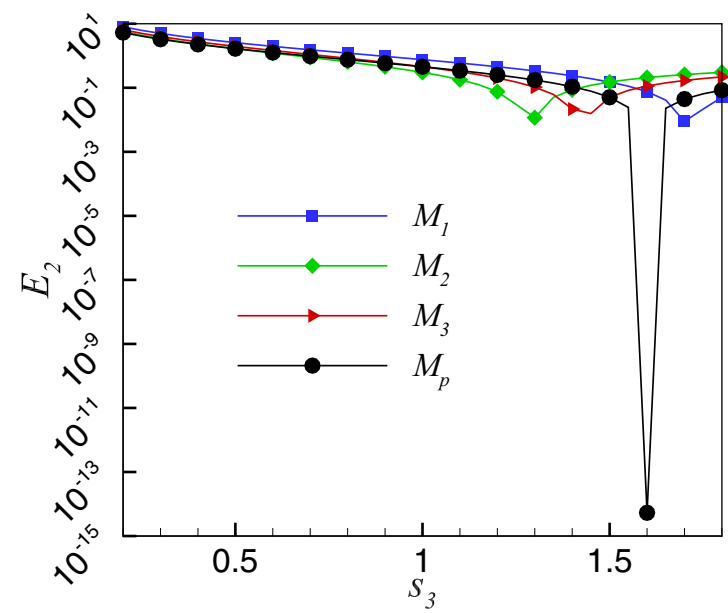

FIG. 1. Comparison of relative errors of different forcing schemes $E_{2}$, as defined in Eq. (27), as a function of $s_{3}$, in steady Poiseuille flow.

parameters are 1.754. As shown in Table I, the relative errors for $M_{1}$ are $O\left(10^{-4}\right)$, which are consistent with the results in Ref. [27]. The small differences among the relative errors here and in Ref. [27] are related to the different choices for $s_{3}$ (the nonslip rule was not considered in Ref. [27]). Compared with these three methods, the relative errors for the present method are 5-6 orders of magnitude smaller, which confirms the conformation of the nonslip rule in the present method. From the discussion in Sec. IIID, it is easy to observe that the error terms in the three methods $M_{1}, M_{2}$, and $M_{3}$ are in a descending order.

\section{B. Steady Taylor-Green flow}

For the two-dimensional steady incompressible flow in a periodic box $N \times N$, if the force field is given by

$$
\mathbf{F}(x, y)=2 v u_{0} \phi^{2}[\cos (\phi x) \cos (\phi y), \sin (\phi x) \sin (\phi y)],
$$

the flow has the following analytical solution:

$$
\begin{aligned}
\mathbf{u}_{a}(x, y) & =u_{0}[\sin (\phi x) \sin (\phi y), \cos (\phi x) \cos (\phi y)], p_{a}(x, y) \\
& =p_{0}+0.25 u_{0}^{2}[\cos (2 \phi x)-\cos (2 \phi y)],
\end{aligned}
$$

where $\phi=2 \pi / N, p_{0}=\rho_{0} c_{s}^{2}$, and $\rho_{0}=1$. The flow is known as steady Taylor-Green flow or four-rolls mill [38], and is characterized by the Reynolds number, $\operatorname{Re}=u_{0} \pi / \nu$. In the simulation, the computational domain is covered by a series of grid nodes, $N / \Delta x=[10,20,40,80]$, with three different conditions of $\operatorname{Re}=[50,100,150]$. To weaken the artificial compressibility, $u_{0}=0.05$ is used in all the cases, $s_{b}$ is given

TABLE I. Relative errors $\left(\times 10^{4}\right)$ of different forcing schemes in steady Poiseuille flow. A series of body forces are considered, and $s_{3}$ is chosen according to the nonslip rule.

\begin{tabular}{lcccc}
\hline \hline$F_{x}$ & $M_{1}$ & $M_{2}$ & $M_{3}$ & $M_{p}$ \\
\hline $1 \times 10^{-6}$ & 2.739 & 2.339 & 1.113 & $1.044 \times 10^{-6}$ \\
$3 \times 10^{-6}$ & 2.739 & 2.339 & 1.113 & $3.173 \times 10^{-6}$ \\
$5 \times 10^{-6}$ & 2.739 & 2.339 & 1.113 & $5.527 \times 10^{-6}$ \\
$7 \times 10^{-6}$ & 2.739 & 2.339 & 1.113 & $7.296 \times 10^{-6}$ \\
\hline \hline
\end{tabular}

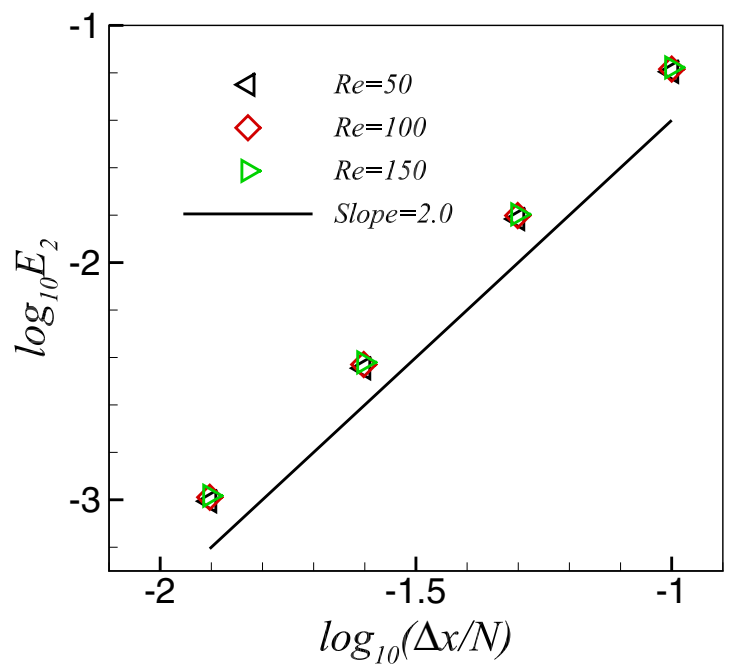

FIG. 2. The second-order accuracy of the new consistent forcing scheme, as measured by the relative errors $E_{2}$, defined in Eq. (27), as a function of grid sizes in steady Taylor-Green flow at $\mathrm{Re}=50,100$, and 150 .

equal to $s_{2}$, while the remaining relaxation parameters are set to unity. The relative error $E_{2}$ is computed from Eq. (27).

The relationship between grid sizes and measured relative errors, $E_{2}$, for the present forcing scheme at different Reynolds numbers is presented in Fig. 2. The slopes at $\mathrm{Re}=50,100$, and 150 are 2.0133, 2.0076, and 2.0068, respectively. This demonstrates that the scheme proposed has second-order accuracy in space. The relative errors for different methods are shown in Table II. It is found that the present scheme achieves the smallest relative error for every grid resolution at every Reynolds number. Due to the discrete equilibrium central moments used in $M_{3}$ (see Eq. (10) in Ref. [39]), some additional errors are introduced into the CLBM, and this effect becomes evident when the mesh size is small. It may be the reason why this method manifests an outlier for the finest grid resolution. Generally, each method presents a second-order convergence rate.

\section{Single static droplet}

To validate the present forcing scheme for a complex force field, we consider the simulations of a static droplet using the Shan-Chen multiphase model [4], which is also known as the pseudopotential approach in the multiphase flow. The interaction force is calculated from an interaction potential $\psi(\mathbf{x})[4]$,

$$
\mathbf{F}=-G \psi(\mathbf{x}) \sum_{i} w_{i} \psi\left(\mathbf{x}+\mathbf{e}_{i} \Delta t\right) \mathbf{e}_{i},
$$

where $G$ is used to control the interaction strength and $w_{i}$ are the weights. When only the nearest-neighbor interactions are considered on the D2Q9 lattice, $w_{i}=1 / 3$ for $\left|\mathbf{e}_{i}\right|^{2}=1$ and $w_{i}=1 / 12$ for $\left|\mathbf{e}_{i}\right|^{2}=2$. The exponential form of the pseudopotential is used, i.e., $\psi(\rho)=\psi_{0} \exp \left(-\rho_{0} / \rho\right)$. Let us denote $\rho_{V}$ and $\rho_{L}$ as the vapor and liquid coexistence densities, respectively. In this study, $\psi_{0}=1, \rho_{0}=1$, and $G=10 / 3$ are used, which leads to $\rho_{V}=0.3675$ and $\rho_{L}=2.783[40,41]$. The simulations are conducted in a periodic box $N \times N=$ 
TABLE II. Relative errors $\left(\times 10^{2}\right)$ and convergence rates $(\mathrm{CR})$ achieved by different forcing schemes at different Reynolds numbers in steady Taylor-Green flow.

\begin{tabular}{|c|c|c|c|c|c|c|c|c|c|c|c|c|}
\hline \multirow[b]{2}{*}{$N / \Delta x$} & \multicolumn{4}{|c|}{$\mathrm{Re}=50$} & \multicolumn{4}{|c|}{$\mathrm{Re}=100$} & \multicolumn{4}{|c|}{$\mathrm{Re}=150$} \\
\hline & $M_{P}$ & $M_{1}$ & $M_{2}$ & $M_{3}$ & $M_{P}$ & $M_{1}$ & $M_{2}$ & $M_{3}$ & $M_{P}$ & $M_{1}$ & $M_{2}$ & $M_{3}$ \\
\hline 10 & 6.3752 & 6.5748 & 6.5522 & 6.3959 & 6.5448 & 6.6456 & 6.6398 & 6.5660 & 6.6482 & 6.7162 & 6.7135 & 6.6698 \\
\hline 20 & 1.5275 & 1.6263 & 1.6051 & 1.5538 & 1.5788 & 1.6283 & 1.6227 & 1.6063 & 1.5974 & 1.6305 & 1.6279 & 1.6261 \\
\hline 40 & 0.3587 & 0.3969 & 0.3782 & 0.3843 & 0.3719 & 0.3961 & 0.3909 & 0.4074 & 0.3796 & 0.3957 & 0.3933 & 0.4145 \\
\hline 80 & 0.0986 & 0.1141 & 0.1037 & 0.1498 & 0.1025 & 0.1109 & 0.1076 & 0.1543 & 0.1040 & 0.1098 & 0.1082 & 0.1560 \\
\hline CR & 2.0133 & 1.9579 & 2.0031 & 1.8264 & 2.0076 & 1.9753 & 1.9896 & 1.8213 & 2.0068 & 1.9848 & 1.9917 & 1.8222 \\
\hline
\end{tabular}

$250 \times 250$. A round droplet of radius $R=50$ is initialized by setting $\rho=\rho_{L}$ in the circle and $\rho=\rho_{V}$ outside the circle. The relaxation parameters are chosen as $s_{b}=s_{2}=1.4$.

The steady-state density contours for varied $s_{3}$ obtained by different forcing schemes are compared in Fig. 3. The inserted dashed circle represents the theoretical location of the droplet. From the first column of Fig. 3 (obtained by the forcing scheme of Premnath et al. [27]), it can be seen that the shape of the droplet is $s_{3}$-dependent and it changes from a noncircular shape to a round one with the increase
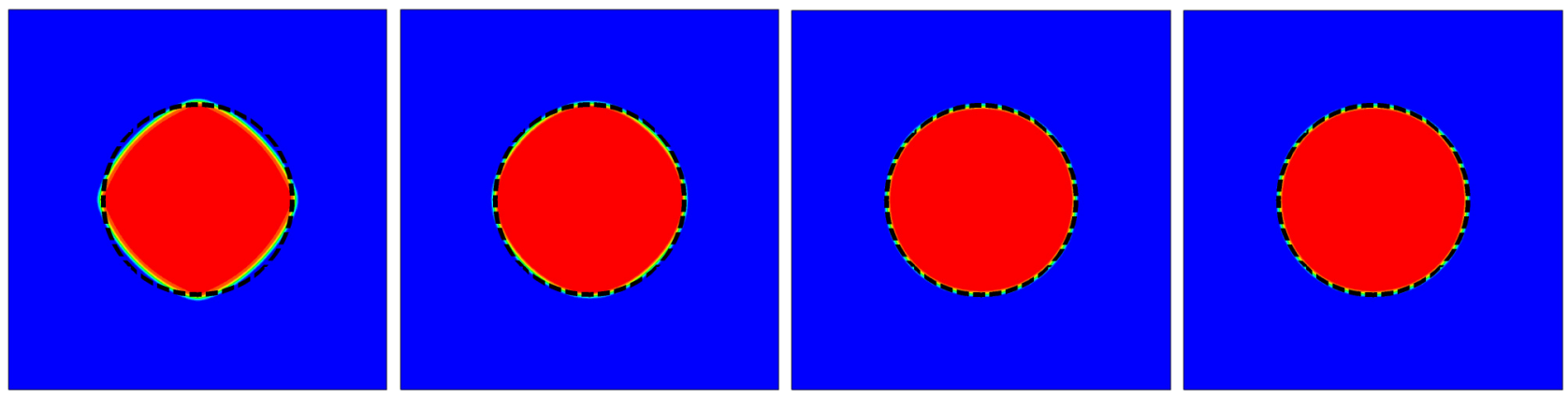

$s_{3}=1.0$
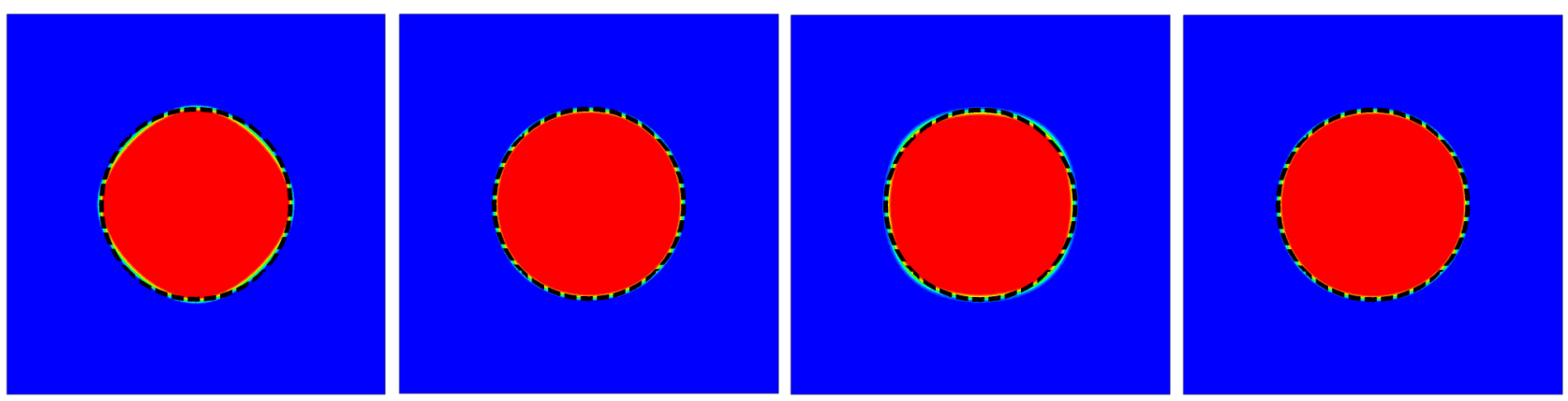

\section{$s_{3}=1.4$}
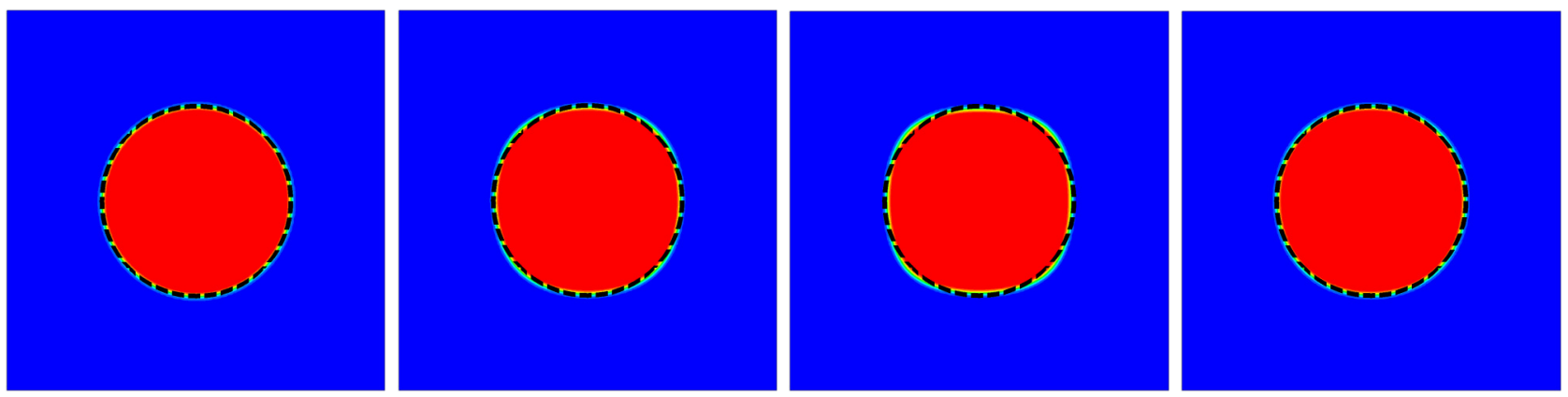

$s_{3}=1.8$

FIG. 3. Steady-state density contours of single static droplet for varied $s_{3}$, achieved by different forcing schemes (from left to right): Premnath et al. [27] (first column), Lycett-Brown and Luo [28] (second column), De Rosis [29] (third column) and the new forcing scheme (fourth column). The additional dashed circle is the theoretical location of the droplet. 


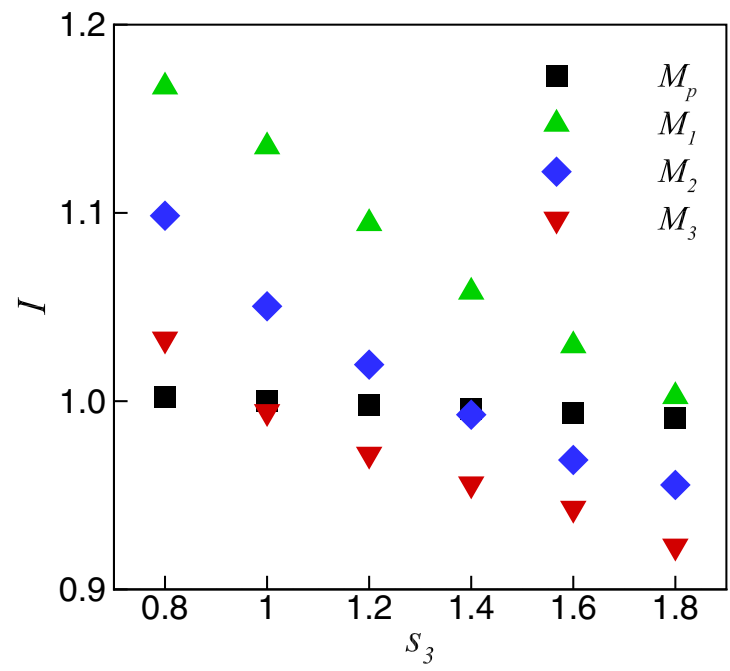

FIG. 4. Isotropy defined by $I=\left|R_{\pi / 4} / R_{0}\right|$, as a function of $s_{3}$ for different forcing schemes for single static droplet simulation.

of $s_{3}$. As discussed in Sec. III A, the removal of high-order terms for the central moments of $\Delta f$ in Ref. [27] introduces inconsistencies with the scheme proposed by Guo et al. [25]. Only when $s_{3}$ is set to be 2.0 , the inconsistency can be eliminated. Although we cannot give the result with $s_{3}=2.0$ (divergent for this simulation), the tendency confirms our argument. Analogously, as discussed in Secs. III B and III C, the inconsistencies in $M_{2}$ and $M_{3}$ can only be eliminated under the conditions of $s_{3}=s_{2}$ and $s_{3}=1.0$, respectively, which is also confirmed in the figure. For the present forcing scheme, the droplet is always in round shape independent of the value of $s_{3}$, as can be seen in the last column of Fig. 3 .

To quantify the inconsistency, we define an isotropy parameter as $I=\left|R_{\pi / 4} / R_{0}\right|$, where the subscript denotes the angle between the $x$ axis and the line along which the radius is measured. From Fig. 4, we can see two clear trends: $I$ in the present forcing scheme is almost independent of $s_{3}$ (the maximal relative variation is less than $1 \%$ ), while $I$ depends on $s_{3}$ apparently in the other methods and the anisotropies can only be eliminated under certain conditions as discussed in Sec. III D.

\section{CONCLUSIONS}

In this study, we present an alternative derivation of the cascaded lattice Boltzmann method. A shift matrix $\mathbf{N}$ is introduced in the derivation, by which the raw moments of the discrete distribution function are shifted to their central moments. This approach puts the MRT LBM and CLBM into a unified general framework and clarifies the relationship between them. Based on this, a new method to incorporate forcing terms into the CLBM is proposed.

The forcing terms are incorporated by means of central moments, which is compatible with the basic ideology of CLBM. According to the definition of the shift matrix $\mathbf{N}$, CLBM degrades into an MRT LBM when $\mathbf{N}$ is a unit matrix. The present forcing scheme retains the property of and degrades into the Guo forcing scheme in the MRT LBM when $\mathbf{N}$ is a unit matrix. Specifically, the present forcing scheme degrades to the original forcing scheme proposed by Guo et al. when all the relaxation parameters are set to be the same. Numerical simulations for several benchmark problems confirm the consistency of the nonslip rule, the second-order accuracy in space, and the property of isotropy for the present forcing scheme. In the meantime, some inconsistences in the previous forcing schemes are also revealed.

The derivation and the method developed are quite intelligible, and no cumbersome operations are involved in the practical implementation. For other discrete velocity models (for example, D3Q15, D3Q19, and D3Q27), the corresponding methods can be developed directly by finding corresponding raw moments sets and obtaining the expressions of $\mathbf{M}, \mathbf{N}$, and $C_{i}$. Further work will demonstrate that the present method can be extended to three dimensions $(3 \mathrm{D})$ readily.

\section{ACKNOWLEDGMENTS}

The authors thank Dr. Chuandong Lin for fruitful discussions. Support from the MOST National Key Research and Development Programme (Project No. 2016YFB0600805) and the Center for Combustion Energy at Tsinghua University is gratefully acknowledged. Supercomputing time on ARCHER is provided by the UK Consortium on Mesoscale Engineering Sciences (UKCOMES) under the UK Engineering and Physical Sciences Research Council Grant No. EP/L00030X/1.

\section{APPENDIX}

The raw moments can be transformed to the discrete DFs through $\mathbf{M}^{-1}$, and the central moments can be shifted to raw moments through $\mathbf{N}^{-1}$,

$$
\left|f_{i}\right\rangle=\mathbf{M}^{-1}\left|T_{i}\right\rangle, \quad\left|T_{i}\right\rangle=\mathbf{N}^{-1}\left|\tilde{T}_{i}\right\rangle
$$

The explicit expressions for $\mathbf{M}^{-1}$ and $\mathbf{N}^{-1}$ are

$$
\mathbf{M}^{-1}=\left[\begin{array}{ccccccccc}
1 & 0 & 0 & -1 & 0 & 0 & 0 & 0 & 1 \\
0 & 1 / 2 & 0 & 1 / 4 & 1 / 4 & 0 & 0 & -1 / 2 & -1 / 2 \\
0 & 0 & 1 / 2 & 1 / 4 & -1 / 4 & 0 & -1 / 2 & 0 & -1 / 2 \\
0 & -1 / 2 & 0 & 1 / 4 & 1 / 4 & 0 & 0 & 1 / 2 & -1 / 2 \\
0 & 0 & -1 / 2 & 1 / 4 & -1 / 4 & 0 & 1 / 2 & 0 & -1 / 2 \\
0 & 0 & 0 & 0 & 0 & 1 / 4 & 1 / 4 & 1 / 4 & 1 / 4 \\
0 & 0 & 0 & 0 & 0 & -1 / 4 & 1 / 4 & -1 / 4 & 1 / 4 \\
0 & 0 & 0 & 0 & 0 & 1 / 4 & -1 / 4 & -1 / 4 & 1 / 4 \\
0 & 0 & 0 & 0 & 0 & -1 / 4 & -1 / 4 & 1 / 4 & 1 / 4
\end{array}\right],
$$


and

$$
\mathbf{N}^{-1}=\left[\begin{array}{ccccccccc}
1 & 0 & 0 & 0 & 0 & 0 & 0 & 0 & 0 \\
u_{x} & 1 & 0 & 0 & 0 & 0 & 0 & 0 & 0 \\
u_{y} & 0 & 1 & 0 & 0 & 0 & 0 & 0 & 0 \\
u_{x}^{2}+u_{y}^{2} & 2 u_{x} & 2 u_{y} & 1 & 0 & 0 & 0 & 0 & 0 \\
u_{x}^{2}-u_{y}^{2} & 2 u_{x} & -2 u_{y} & 0 & 1 & 0 & 0 & 0 & 0 \\
u_{x} u_{y} & u_{y} & u_{x} & 0 & 0 & 1 & 0 & 0 & 0 \\
u_{x}^{2} u_{y} & 2 u_{x} u_{y} & u_{x}^{2} & u_{y} / 2 & u_{y} / 2 & 2 u_{x} & 1 & 0 & 0 \\
u_{y}^{2} u_{x} & u_{y}^{2} & 2 u_{x} u_{y} & u_{x} / 2 & -u_{x} / 2 & 2 u_{y} & 0 & 1 & 0 \\
u_{x}^{2} u_{y}^{2} & 2 u_{x} u_{y}^{2} & 2 u_{y} u_{x}^{2} & u_{x}^{2} / 2+u_{y}^{2} / 2 & u_{y}^{2} / 2-u_{x}^{2} / 2 & 4 u_{x} u_{y} & 2 u_{y} & 2 u_{x} & 1
\end{array}\right]
$$

[1] Y.-H. Qian, S. Succi, and S. A. Orszag, Annu. Rev. Comput. Phys. 3, 195 (1995).

[2] S. Chen and G. D. Doolen, Annu. Rev. Fluid Mech. 30, 329 (1998).

[3] S. Succi, The Lattice Boltzmann Equation: For Fluid Dynamics and Beyond (Oxford University Press, Oxford, 2001).

[4] X. Shan and H. Chen, Phys. Rev. E 47, 1815 (1993).

[5] Z. Guo and C. Shu, Lattice Boltzmann Method and Its Applications in Engineering, Vol. 3 (World Scientific, Singapore, 2013).

[6] W. Gong, Y. Zu, S. Chen, and Y. Yan, Sci. Bull. 62, 136 (2016).

[7] C. Lin, A. Xu, G. Zhang, and Y. Li, Combust. Flame 164, 137 (2016).

[8] C. Lin, A. Xu, G. Zhang, Y. Li, and S. Succi, Phys. Rev. E 89, 013307 (2014).

[9] Q. Li, K. H. Luo, Q. Kang, Y. He, Q. Chen, and Q. Liu, Prog. Energy Combust. Sci. 52, 62 (2016).

[10] Y. Qian, D. d'Humières, and P. Lallemand, Europhys. Lett. 17, 479 (1992).

[11] D. d'Humieres, Prog. Astronaut. Aeronaut. 159, 450 (1994).

[12] M. Geier, A. Greiner, and J. G. Korvink, Phys. Rev. E 73, 066705 (2006).

[13] P. Lallemand and L.-S. Luo, Phys. Rev. E 61, 6546 (2000).

[14] D. Lycett-Brown and K. H. Luo, Phys. Rev. E 94, 053313 (2016).

[15] L. Fei and K. H. Luo, arXiv:1610.07114 (2016).

[16] I. Ginzburg, Adv. Water Res. 28, 1171 (2005).

[17] I. Ginzburg, F. Verhaeghe, and D. d'Humieres, Commun. Comput. Phys. 3, 427 (2008).

[18] S. Ansumali, I. V. Karlin, and H. C. Öttinger, Europhys. Lett. 63, 798 (2003).

[19] S. Ansumali and I. V. Karlin, Phys. Rev. E 62, 7999 (2000).
[20] X. He, S. Chen, and R. Zhang, J. Comput. Phys. 152, 642 (1999).

[21] J. M. Buick and C. A. Greated, Phys. Rev. E 61, 5307 (2000).

[22] A. Ladd and R. Verberg, J. Stat. Phys. 104, 1191 (2001).

[23] X. He, X. Shan, and G. D. Doolen, Phys. Rev. E 57, R13 (1998).

[24] X. He, S. Chen, and G. D. Doolen, J. Comput. Phys. 146, 282 (1998).

[25] Z. Guo, C. Zheng, and B. Shi, Phys. Rev. E 65, 046308 (2002).

[26] Z. Guo and C. Zheng, Int. J. Comput. Fluid Dynam. 22, 465 (2008).

[27] K. N. Premnath and S. Banerjee, Phys. Rev. E 80, 036702 (2009).

[28] D. Lycett-Brown and K. H. Luo, Comput. Math. Appl. 67, 350 (2014).

[29] A. De Rosis, Phys. Rev. E 95, 023311 (2017).

[30] P. Asinari, Phys Rev E 78, 016701 (2008).

[31] Q. Liu, Y.-L. He, D. Li, and Q. Li, Int. J. Heat Mass Transfer 102, 1334 (2016).

[32] I. Ginzburg and D. d'Humières, Phys. Rev. E 68, 066614 (2003).

[33] A. Kupershtokh, D. Medvedev, and D. Karpov, Comput. Math. Appl. 58, 965 (2009).

[34] H. Huang, M. Krafczyk, and X. Lu, Phys. Rev. E 84, 046710 (2011).

[35] Q. Li, K. H. Luo, and X. J. Li, Phys. Rev. E 86, 016709 (2012).

[36] D. Lycett-Brown and K. H. Luo, Phys. Rev. E 91, 023305 (2015).

[37] R. K. Freitas, A. Henze, M. Meinke, and W. Schröder, Comput. Fluids 47, 115 (2011).

[38] G. Taylor, Proc. R. Soc. London A 146, 501 (1934).

[39] A. De Rosis, Europhys. Lett. 116, 44003 (2017).

[40] Z. Yu and L.-S. Fan, J. Comput. Phys. 228, 6456 (2009).

[41] Q. Li, P. Zhou, and H. J. Yan, Phys. Rev. E 94, 043313 (2016). 